\title{
Design and Development of an Optical Path Difference Scan Mechanism for Fourier Transform Spectrometers using High Displacement RAINBOW Actuators
}

\author{
Stephanie A. Wise ${ }^{\mathrm{a}}$, Robin C. Hardy ${ }^{\mathrm{a}}$, and David E. Dausch \\ NASA-Langley Research Center, Hampton, VA 23681 \\ 'National Research Council, NASA-Langley Research Center, Hampton, VA 23681
}

\begin{abstract}
A new piezoelectric drive mechanism has been developed for optical translation in space-based spectrometer systems. The mechanism utilizes a stack of RAINBOW high displacement piezoelectric actuators to move optical components weighing less than 250 grams through a one centimeter travel. The mechanism uses the direct motion of the piezoelectric devices, stacked such that the displacement of the individual RAINBOW actuators is additive. A prototype device has been built which utilizes 21 RAINBOWs to accomplish the necessary travel. The mechanism weighs approximately 0.6 kilograms and uses less than 2 Watts of power at a scanning frequency of $0.5 \mathrm{Hertz}$, significantly less power than that required by state-of-the-art motor systems.
\end{abstract}

Keywords: Optical translation, Fourier transform spectrometers, high displacement actuators, RAINBOWs, actuator stacks

\section{INTRODUCTION}

The next generation of space instruments designed by NASA will be smaller, lighter, and need less power to operate in order to reduce the launch and operations costs associated with undertaking space or Earth science missions. In order to meet these requirements, advanced technologies and new materials will be needed to produce significant improvements over state-of-the-art approaches. As a result, component-level investigations are underway to determine the extent to which NASA instruments can be modified to meet future mission goals while also reducing cost and maintaining science performance.

One type of instrument under development is a Fourier Transform Spectrometer (FTS) which uses Michelson interferometry to measure the far infrared emission of the Earth's atmosphere, thus determining science parameters such as temperature, cloud composition, and surface weather and climate data. ${ }^{1}$ During instrument operation, two mirrors are translated simultaneously in opposite directions, continuously varying the optical path difference to create interference patterns. The distance of travel required for these mirrors ranges from one to six centimeters of motion, depending on the system parameters.

Currently, spacecraft spectrometer instruments use brushless DC motors for optics translation. However, motor-driven systems can be relatively large, heavy components which consume 10-15 W of power during operation. One desirable alternative is to create a solid-state, piezoelectric drive mechanism which provides direct, high precision optical translation at reduced mass and power.

Conventional piezoelectric materials, such as multilayer stack actuators, produce large forces but very small displacements (i.e., microns of motion). ${ }^{2}$ These actuators have been demonstrated for space 
applications such as on-orbit focusing of mirrors, ${ }^{3}$ which requires precise movement of individual actuators. In order to achieve larger motions, inchworm motors are typically developed. ${ }^{4-7}$ These devices employ piezoelectrics to alternatively clamp and translate a shaft, thus realizing large displacements by producing multiple steps at high frequencies. Although demonstrating high resolution and the ability to produce continuous motion through cycle repetition, these devices are generally not suited for spectrometer applications where linearity of motion is critical. Due to minor shifting of the shaft position during a clamp change, "jerking" of the output motion is a consequence which has not been possible to eliminate.

High displacement piezoelectric actuators, such as the recently discovered RAINBOW (Reduced And INternally Biased Oxide Wafer) device, ${ }^{8}$ offer a unique alternative to conventional piezoelectrics for optical translation. RAINBOWs are monolithic unimorph-type actuators produced by chemically reducing a portion of a high-lead-containing piezoelectric ceramic such as PZT (i.e., lead zirconate titanate). Reduction is performed by placing the ceramic on a graphite block at an elevated temperature (e.g., $975^{\circ} \mathrm{C}$ ) for a predetermined period of time sufficient to remove oxygen from one side of the piezoelectric wafer. Thus, an oxygen-deficient, highly-metallic, reduced layer is created within the monolithic part.

The reduced layer in the RAINBOW behaves in a similar manner to the metal foil used in a conventional unimorph, constricting lateral motion and amplifying displacement through the thickness of the piezoelectric.' Additionally, during cooling, the thermal expansion differences between the reduced layer and the piezoelectric layer in the RAINBOW create internal stress in the piezoelectric portion, further amplifying displacement and producing a domed structure, with the piezoelectric in compression.

Single RAINBOW elements can produce displacements of several hundred microns depending on the actuator size, a substantial increase compared to the strain capability of the starting piezoelectric. Additionally, individual RAINBOW devices can be stacked together to produce even greater displacements. While the large generative forces characteristic of multilayer stacks are not produced, individual RAINBOWs can sustain loads in excess of $1 \mathrm{~kg}$, making them suitable for optical translation mechanisms. Finally, the power consumption of a RANBOW stack is extremely low compared to multilayer stack actuators, offering an additional advantage for space applications.

Based on the unique properties of RAINBOW actuators in the present study, a direct motion piezoelectric scan mechanism for a typical FTS instrument has been designed, fabricated, and tested. This mechanism employs a stack of RAINBOW actuators to continuously cycle an optical component through a onecentimeter stroke. The mechanism design, mass and power consumption characteristics, and displacement performance will be discussed.

\section{MECHANISM DESIGN AND TESTING}

\subsection{Mechanism requirements}

The requirements for the optical translation mechanism were derived based on the design of a small-scale FTS instrument. Two mechanisms are required per instrument, with each translating one interferometer mirror. Each mirror is required to travel one centimeter with the mirror weighing no more than $250 \mathrm{~g}$. Although the scan rate of the instrument is dependent on the orbit and measurement parameters, a typical scanning frequency of $1 \mathrm{~Hz}$ was used for displacement performance testing. These requirements are summarized in Table I. 
Table I. Scan mechanism requirements.

\begin{tabular}{|c|c|}
\hline Parameter & Requirement \\
\hline Displacement distance & $1 \mathrm{~cm}$ \\
\hline Mirror mass & $\sim 250 \mathrm{~g}$ \\
\hline Frequency of scan & 0.2 to $1 \mathrm{~Hz}$ \\
\hline
\end{tabular}

\subsection{Mechanism design}

The one-centimeter stroke requirement for the FTS instrument necessitated the use of a stack of RAINBOWs as opposed to a single actuator. One goal of this study was to determine the optimum size and number of the RAINBOWs required for the stack. Therefore, a mechanism was designed which allowed for flexibility in interchanging the number of RAINBOWs within the stack, as well as the diameter of the RAINBOW parts. The actuator core was designed to accommodate RAINBOWs up to $5.08 \mathrm{~cm}$ in diameter. Aluminum top and bottom plates of the support structure were approximately 9 centimeters in diameter, connected by three aluminum posts. The inner core of actuators consisted of a housing to contain the actuator elements with slots on either side to accommodate the positive and negative lead wires for the RAINBOWs in the stack. In the prototype unit, individual housings were fabricated for two representative actuator diameters, namely 2.54 and $5.08 \mathrm{~cm}$. Because the number of parts needed at each size was not yet known, the inner core units were fabricated at a maximum expected height of $7.6 \mathrm{~cm}$, and Teflon inserts were used to fill the extra space when shorter stacks were tested.

The mirror used for the prototype unit was a corner cube retroreflector approximately $6.4 \mathrm{~cm}$ on the longest side. The mirror was held in place with an adjustable frame which rested on the actuator core via a rod. As a result, direct translation of the optic by means of the RAINBOW stack was achieved. A schematic of the mechanism illustrating the mirror, support structure, RAINBOW stack, and slot for the lead wires is shown in Figure 1.

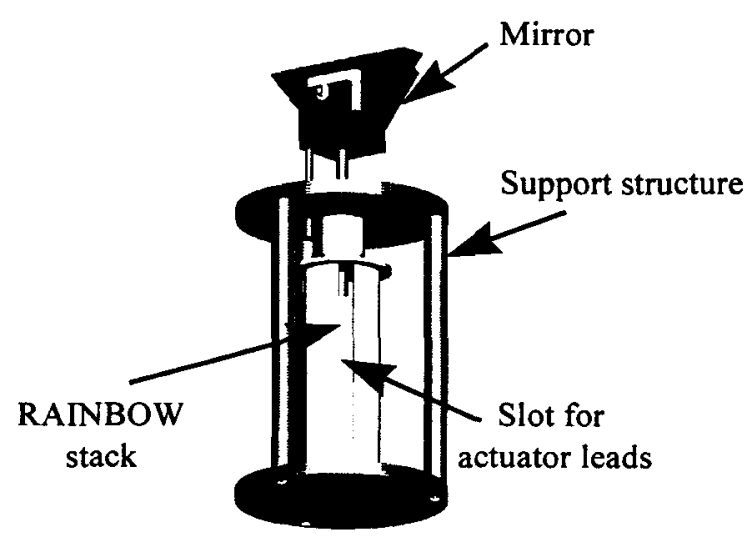

Figure 1. RAINBOW scan mechanism schematic.

\subsection{Actuator fabrication and stack assembly}

RAINBOW actuators were fabricated from two piezoelectric compositions, namely PZT-5A and PZT-5H, at diameters of 2.54 and $5.08 \mathrm{~cm}$. The $2.54-\mathrm{cm}$ diameter parts were 380 microns thick, while the 5.08 centimeter diameter parts were 500 microns thick. At the larger diameters, thinner RAINBOWs were found to be "saddle-shaped" as described by Haertling. ${ }^{10}$ Because of the difficulty in stacking saddled devices, thicker piezoelectric wafers were used to produce the 5.08-cm diameter RAINBOWs. 
The reduced layers in the RAINBOWs were $-35-50 \%$ of the total thickness which provided optimum displacement performance. " After reduction, the top and bottom surfaces of the RAINBOWs were electroded with silver epoxy. Copper tabs ( 0.1 millimeters thick) with soldered leads were then attached to the RAINBOWs, and the parts were coated with a dielectric sealant (GC Electronics, EL-5012 polyester resin).

Once the RAINBOW actuators were fabricated, stacks were produced by placing the individual elements in clamshell configurations, which were electrically connected in parallel. Details regarding the stacking configuration used and the displacement performance of smaller stacks of RAINBOW devices have been reported elsewhere. ${ }^{12}$

\subsection{Testing}

The displacement characteristics of the individual RAINBOW actuators were measured using a linear variable displacement transducer (LVDT). Because of the difference in thickness of the piezoelectric parts used in this study, a constant electric field strength of $\pm 16 \mathrm{kV} / \mathrm{cm}$ was used for all tests. Both free displacement (i.e., no load) and displacement under a $250 \mathrm{~g}$ static load were measured. Based on average values from this data, the number of RAINBOWs required to produce the necessary motion was estimated.

Next, the mass and power values for the different RAINBOW stacks were determined. The scan mechanism components, including the RAINBOW stacks, were weighed, and the total mechanism mass determined. Additionally, the capacitance values of the individual RAINBOWs produced from the different sizes and compositions were measured at $1 \mathrm{kHz}$ and $500 \mathrm{mV}$ using an LCR meter. The capacitance of the stack was then determined by multiplying the average capacitance per part by the number of parts needed to achieve $1 \mathrm{~cm}$. The power required to operate each RAINBOW stack was then calculated using the following relationship: ${ }^{13}$

$$
\mathrm{P}=\pi \cdot \mathrm{f} \cdot \mathrm{C} \cdot \mathrm{V}^{2},
$$

where $P$ is the power $(W), f$ is the frequency $(H z), C$ is the stack capacitance $(F)$, and $V$ is the voltage $(V)$.

Based on the information obtained, a stack of $5.08-\mathrm{cm}$ diameter RAINBOW actuators was produced in order to demonstrate the one centimeter stroke. Additional actuators were added as needed to offset small losses in displacement due to mechanical slippage along the RAINBOW interfaces.

\section{EXPERIMENTAL RESULTS}

\subsection{RAINBOW actuator displacement}

The average displacement values measured at $\pm 16 \mathrm{kV} / \mathrm{cm}$ for the individual RAINBOWs are shown in Table II. The estimated number of actuators required for one centimeter of motion is listed. This value was used to determine the mass of the RAINBOW stacks.

Table II. Average displacement values for individual RAINBOW actuators.

\begin{tabular}{|c|c|c|c|c|}
\hline $\begin{array}{c}\text { Piezoelectric } \\
\text { composition }\end{array}$ & $\begin{array}{c}\text { Part Diameter, } \\
\mathbf{c m}\end{array}$ & $\begin{array}{c}\text { Avg. free } \\
\text { displacement, } \mathbf{~ m m}\end{array}$ & $\begin{array}{c}\text { Avg. disp. under } \\
\text { 250 g load, } \mathbf{~ m m}\end{array}$ & $\begin{array}{c}\text { Estimated \# Parts } \\
\text { for } \mathbf{1} \mathbf{~ c m ~ m o t i o n ~}\end{array}$ \\
\hline PZT-5A & 2.54 & 0.38 & 0.38 & 26 \\
\hline PZT-5A & 5.08 & 0.67 & 0.64 & 16 \\
\hline PZT-5H & 2.54 & 0.23 & 0.21 & 48 \\
\hline PZT-5H & 5.08 & 0.30 & 0.29 & 35 \\
\hline
\end{tabular}




\subsection{Mass of the scan mechanism}

The scan mechanism, including the prototype mirror, weighed approximately $.39 \mathrm{~kg}$ prior to the addition of the actuator core. The size and mass of the core were dependent on the diameter of the RAINBOW and the composition of the starting piezoelectric ceramic (i.e., PZT-5A or PZT-5H). Using the estimated number of RAINBOWs needed to produce the required motion, the mass of the stacks was measured, and the effect of the actuator type on the total mechanism mass was determined. The mass data are shown in Table III. Mass values for the actuator stacks reflect the masses of both the piezoelectrics and the associated lead wires. The lead wires comprise a significant portion $(-50 \%)$ of the stack mass for the $2.54-\mathrm{cm}$ diameter stacks, while comprising only $\sim 15 \%$ of the mass for the larger stacks.

Table III. Mass of the actuator stacks, including lead wires.

\begin{tabular}{|c|c|c|}
\hline RAINBOW stack & Actuator stack mass, $\mathrm{kg}$ & Total mechanism mass, $\mathbf{k g}$ \\
\hline $2.54-\mathrm{cm}$ PZT-5A RAINBOWs & 0.08 & 0.47 \\
\hline 5.08 -cm PZT-5A RAINBOWs & 0.15 & 0.54 \\
\hline $2.54-\mathrm{cm}$ PZT-5H RAINBOWs & 0.14 & 0.53 \\
\hline 5.08 -cm PZT-5H RAINBOWs & 0.32 & 0.71 \\
\hline
\end{tabular}

\subsection{Power consumption}

The capacitance of the individual RAINBOWs was measured to determine the effect of part diameter and piezoelectric composition on the power consumed. The voltage level used for all calculations was determined based on the electric field of $\pm 16 \mathrm{kV} / \mathrm{cm}$. Capacitance data, in $\mathrm{nF}$, and power consumption at $0.2,0.5$, and $1 \mathrm{~Hz}$ are provided in Table IV.

Table IV. Power consumption of various RAINBOW stacks.

\begin{tabular}{|c|c|c|c|c|c|c|}
\hline $\begin{array}{c}\text { RAINBOW } \\
\text { type }\end{array}$ & $\begin{array}{c}\text { Capacitance } \\
\text { per part, } \\
\mathbf{n F}\end{array}$ & $\begin{array}{c}\text { Estimated } \\
\text { \# parts } \\
\text { required }\end{array}$ & $\begin{array}{c}\text { Stack } \\
\text { Capacitance } \\
\mathbf{n F}\end{array}$ & $\begin{array}{c}\text { Power at } \\
\mathbf{0 . 2 ~ H z ,} \\
\text { Watts }\end{array}$ & $\begin{array}{c}\text { Power at } \\
\mathbf{0 . 5} \mathbf{~ H z}, \\
\text { Watts }\end{array}$ & $\begin{array}{c}\text { Power at } \\
\mathbf{1 ~ H z ,} \\
\text { Watts }\end{array}$ \\
\hline $\begin{array}{c}2.54-\mathrm{cm} \\
\text { diam. } \\
\text { PZT-5A }\end{array}$ & 17.6 & 26 & 458 & 0.13 & 0.32 & 0.65 \\
\hline $\begin{array}{c}5.08-\mathrm{cm} \\
\text { diam. } \\
\text { PZT-5A }\end{array}$ & 54.1 & 16 & 866 & 0.54 & 1.36 & 2.72 \\
\hline $\begin{array}{c}2.54-\mathrm{cm} \\
\text { diam. } \\
\text { PZT-5H }\end{array}$ & 25.8 & 48 & 1238 & 0.35 & 0.87 & 1.75 \\
\hline $\begin{array}{c}5.08-\mathrm{cm} \\
\text { diam. } \\
\text { PZT-5H }\end{array}$ & 97.4 & 35 & 3409 & 2.14 & 5.35 & 10.71 \\
\hline
\end{tabular}

\subsection{RAINBOW stack performance}

Based on the results of the mass and power measurements, PZT-5A RAINBOWs were selected for integration into the scan mechanism. Minimizing the number of parts used was highly desirable to reduce both the number of mechanical interfaces in the stack and the number of lead wires required. As such, the $5.08-\mathrm{cm}$ diameter RAINBOWs were chosen for further testing. 
The RAINBOW stack was constructed by using an odd number of devices so that the bottom element of the stack was concave-up. Initially, a stack of 17 RAINBOWs was produced and the vertical motion tested at $+16 \mathrm{kV} / \mathrm{cm}$ under a $250 \mathrm{~g}$ load. The stack produced a total stroke of 0.87 centimeters, approximately $13 \%$ less than expected from Table II. To compensate for this mechanical loss, four additional RAINBOWs were added to the stack to increase the displacement performance.

As shown in Figure 2, the stack of 21 RAINBOWs was found to demonstrate the required one centimeter of travel at $1 \mathrm{~Hz}$. The mass of this stack was approximately 200 grams, resulting in a total mechanism mass of $0.6 \mathrm{~kg}$. The power consumed by the stack was $0.71,1.79$, and $3.57 \mathrm{~W}$ when operated at $\pm 16 \mathrm{kV} / \mathrm{cm}$ at frequencies of $0.2,0.5$, and $1 \mathrm{~Hz}$, respectively.

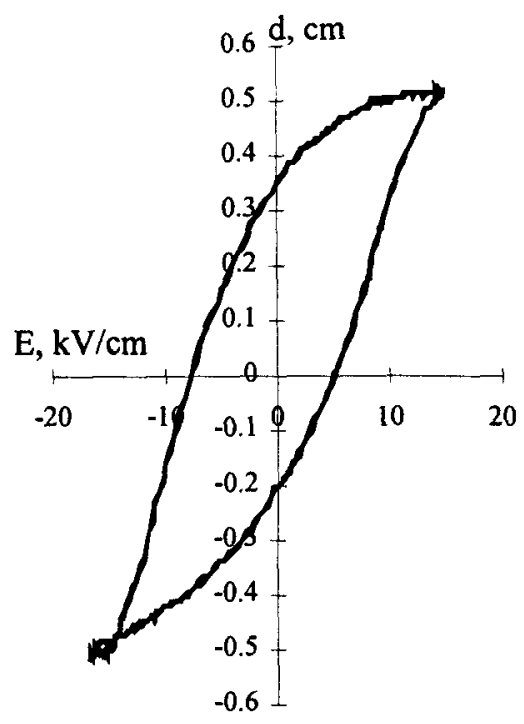

Figure 2. Displacement (d) vs. Electric field (E) performance at $1 \mathrm{~Hz}$ for piezoelectric stack containing 21 RAINBOW actuators produced from $5.08-\mathrm{cm}$ diameter PZT-5A ceramics.

\section{DISCUSSION}

The RAINBOW stacks produced from PZT-5A ceramics exhibited approximately twice the displacement of those produced from PZT $-5 \mathrm{H}$ at $\pm 16 \mathrm{kV} / \mathrm{cm}$. Additionally, the $250 \mathrm{~g}$ load applied to the stacks had no significant effect on the displacement performance. A complete discussion of the displacement properties of PZT-5A and PZT-5H RAINBOWs and RAINBOW stacks is available elsewhere ${ }^{12}$.

Because PZT-5A RAINBOWs produced greater displacements than PZT-5H components, fewer parts were required for the prototype mechanism. For example, 35 5.08-centimeter diameter PZT-5H parts were estimated to produce one centimeter of travel, whereas only 16 PZT-5A components of the same size were estimated to generate this motion. Additionally, the use of PZT-5A RAINBOWs reduced the total mass and power requirements for the stack as compared to the PZT-5H RAINBOWs.

The PZT-5A RAINBOW stacks weighed less than the PZT-5H stacks, based on the required number of RAINBOW parts to produce the necessary stroke. The smaller diameter PZT-5A RAINBOW stack weighed the least, approximately $80 \mathrm{~g}$, with the 5.08-centimeter diameter PZT-5A stack weighing approximately $150 \mathrm{~g}$. The total mechanism weight in both cases was approximately $0.5 \mathrm{~kg}$, which is comparable to that of an electric motor. 
The PZT-5A RAINBOW stacks also consumed significantly less power than the PZT-5H RAINBOW stacks. In the case of the larger diameter PZT-5A RAINBOWs, $0.5-2.7 \mathrm{~W}$ of power was required, depending on the frequency of operation. For a similarly sized PZT-5H RAINBOW stack, $10.7 \mathrm{~W}$ of power was needed at the maximum frequency considered. Due to the stringent limitations on power consumption in spacecraft systems, the PZT-5A materials offer a significant advantage over comparable electric motors which can require up to $15 \mathrm{~W}$ of power.

Based on these results, $5.08-\mathrm{cm}$ diameter PZT-5A RAINBOWs were selected for integration into the scanning mechanism. A stack of 21 RAINBOWs was found to produce the necessary one centimeter of stroke. The slight reduction in displacement performance of the stack as compared to the individual actuators has been found to be the result of mechanical slippage at the edges of the RAINBOWs in the stack, preventing all of the parts from contributing completely to the total motion. The final mechanism weighed approximately $0.6 \mathrm{~kg}$ and consumed less than $2 \mathrm{~W}$ of power at $0.5 \mathrm{~Hz}$. As compared to conventional brushless motors, the piezoelectric mechanism consumed significantly less power at comparable mass, offering a feasible solid-state alternative to electric motors.

\section{SUMMARY}

A comparison of the displacement, power consumption, and mass of PZT-5A and PZT-5H RAINBOW stacks was performed. RAINBOWs produced from PZT-5A exhibited significantly greater displacements. As a result, fewer devices were required to produce the necessary one-centimeter stroke. Based on the estimated number of parts required, the PZT-5A RAINBOW stacks weighed less than the PZT-5H stacks. PZT-5A RAINBOW stacks also consumed less power than stacks produced from PZT-5H RAINBOWs, based on the difference in capacitance between the two compositions as well as the number of devices required. Because fewer parts were needed when larger diameter RAINBOWs were used, the $5.08-\mathrm{cm}$ diameter PZT-5A RAINBOWs were selected for integration into the scan mechanism. Testing of the piezoelectric mechanism revealed that one centimeter of total stroke could be achieved by using 21 RAINBOW parts when operated at $\pm 16 \mathrm{kV} / \mathrm{cm}$ under a $250 \mathrm{~g}$ load. The power consumed at $0.5 \mathrm{~Hz}$ was less than $2 \mathrm{~W}$, considerably less than that of a comparable motor system.

\section{ACKNOWLEDGEMENTS}

The authors gratefully acknowledge Dr. Matthew Hooker of Lockheed Martin Engineering and Sciences for the production and characterization of the RAINBOW actuators used for prototype fabrication and testing. The authors also acknowledge Mr. Dwayne Hinton and Dr. Ira Nolt of NASA-Langley Research Center and Mr. Mark Abrams of ITT Aerospace for providing valuable information on the operational requirements of space-based spectrometer instruments.

\section{REFERENCES}

1. R. Holdaway, "U.K. Instruments for Mission to Planet Earth," Space Technology, 13 [6], pp. 561-7, 1993.

2. S. Takahashi, "Longitudinal Mode Multilayer Piezoceramic Actuators," Ceramic Bulletin, 65, pp. 1156-7, 1986.

3. M.A. Ealey, "Active and Adaptive Optical Components: A General Overview," Proceedings of the ADPA/AIAA/ASME/SPIE Conference on Active Materials and Adaptive Structures, November 4-8, 1991, Alexandria, VA, ed. by G.J. Knowles, pp. 781-3. 
4. J.E. Miesner and J.P. Teter, "Piezoelectric/Magnetostrictive Resonant Inchworm Motor," Proceedings of the 1994 SPIE Smart Materials and Structures Conference - Smart Structures and Intelligent Systems, February 14-16, 1994, Orlando, FL, ed. by N.W. Haygood, pp. 520-7.

5. L.D. Jones, D.V. Newton, and E. Garcia, "Adaptive Devices for Precise Position Control," Proceedings of the 1993 SPIE Smart Materials and Structures Conference - Smart Structures and Intelligent Systems, February 1-4, 1993, Albuquerque, NM, ed. by N.W. Haygood and G.J. Knowles, pp. 648-59.

6. B. Zhang and Z.Q. Zhu, "Design of an Inchworm-type Linear Piezomotor," Proceedings of the 1994 SPIE Smart Materials and Structures Conference - Smart Structures and Intelligent Systems, February 14-16, 1994, Orlando, FL, ed. by N.W. Haygood, pp. 528-39.

7. R.Venkataraman, P.S. Krishnaprasad, and W.P. Dayawansa, "Smart Motor Concept Based on Piezoelectric-Magnetostrictive Resonance," Proceedings of the 1995 SPIE Smart Materials and Structures Conference - Smart Structures and Integrated Systems, February 27-March 3, 1995, San Diego, CA, ed. by I. Chopra, pp. 763-70.

8. G.H. Haertling, "Chemically Reduced PLZT Ceramics for Ultra-High Displacement Actuators," Ferroelectrics, 154, pp. 101-6, 1994.

9. C.P. Germano, "Flexure Mode Piezoelectric Transducers," IEEE Transactions on Audio and Electroacoustics, AU-19 [1], pp. 6-12, 1971.

10. G. H. Haertling, "RAINBOWS and Ferrofilms - Smart Materials for Hybrid Microelectronics," Hybrid Microelectronic Materials: Ceramic Transactions, ed. by K.M. Nair and V.N. Shukla, Volume 68, pp. 71-96, American Ceramic Society, Westerville, Ohio, 1996.

11. S.A. Wise, "Process Optimization of RAINBOW High Displacement Piezoelectric Actuators," presented at the Fall Meeting of the American Ceramic Society, San Antonio, TX, October 30 November 2, 1996.

12. M.W. Hooker, "Properties and Performance of RAINBOW Piezoelectric Actuators," these proceedings.

13. "Piezo Guide: Piezo Positioning Technology Part 1", Catalogue PZ 36E, in Products for Micropositioning, Physik Instrumente, Catalogue 108-12/90.14. 
